\title{
L-tetrahydropalmatine reduces oxaliplatin-induced neurotoxicity by selectively inhibiting the transporter-mediated uptake in the dorsal root ganglion
}

\author{
Yaodong $\mathrm{Yi}^{1}$, Liping $\mathrm{Li}^{1}$, Feifeng Song ${ }^{1}$, Mingyang Chen ${ }^{1}$, Yingchun Chen ${ }^{1}, \mathrm{Ping}^{1}{ }^{1}$, \\ Shixin $\mathrm{Ni}^{1}$, Hui Zhou ${ }^{2}$, Su Zeng ${ }^{2}$, and Huidi Jiang ${ }^{3}$ \\ ${ }^{1}$ Zhejiang University, College of Pharmaceutical Sciences 866 Yuhangtang Road, Hangzhou \\ 310058, China Hangzhou, Zhejiang, CN 310058 (86)-571-88208404 Hangzhou, CN \\ Hangzhou, CN \\ ${ }^{2}$ Zhejiang University, Hangzhou, Zhejiang P.R. China \\ ${ }^{3}$ Zhejiang University
}

May 5, 2020

\begin{abstract}
Background and Purpose Oxaliplatin (OXA) is a third-generation anti-tumour platinum drug; however, the high accumulation of OXA in the dorsal root ganglia (DRG) induces severe peripheral neurotoxicity, which limits its application. This study aims to confirm the role of OCT2, OCTN1, and OCTN2 in the transcellular transport of OXA and to explore whether L-tetrahydropalmatine (L-THP) would selectively inhibit the uptake transporters and subsequently alleviate OXA-induced peripheral neurotoxicity. Experimental Approaches Transporter-transfected MDCK cells, primary DRG cells and tumour cell lines were utilized in vitro studies. OXA-induced chronic peripheral neurotoxicity mice and tumour-bearing nude mice were used in vivo studies. Key Results OCT2 and OCTN1 but not OCTN2 were involved in the uptake of OXA; OCT2 played the most important role. L-THP reduced the cytotoxicity and cellular accumulation of OXA in a concentration-dependent manner in MDCK-hOCT2, MDCK-hOCTN1, and rat primary DRG cells but did not affect its efflux from MDCK-hMRP2 cells. Furthermore, L-THP attenuated OXA-induced peripheral neurotoxicity and reduced the platinum concentration in the DRG in mice in a dose-dependent manner. L-THP did not reduce the platinum concentration in the tumours and did not impair the antitumour efficacy of OXA in HT29 tumour-bearing nude mice nor in tumour cells (HT29 and SW620 cells). Conclusion and Implications OCT2 and OCTN1, especially OCT2, contribute to OXA uptake in the DRG. L-THP attenuates OXA-induced peripheral neurotoxicity via inhibiting OCT2 and OCTN1 but without impairing the antitumour efficacy of OXA. L-THP is a potential candidate drug to attenuate OXA-induced peripheral neurotoxicity.
\end{abstract}

\section{Background and Purpose}

Oxaliplatin (OXA) is a third-generation anti-tumour platinum drug; however, the high accumulation of OXA in the dorsal root ganglia (DRG) induces severe peripheral neurotoxicity, which limits its application. This study aims to confirm the role of OCT2, OCTN1, and OCTN2 in the transcellular transport of OXA and to explore whether L-tetrahydropalmatine (L-THP) would selectively inhibit the uptake transporters and subsequently alleviate OXA-induced peripheral neurotoxicity.

\section{Experimental Approaches}

Transporter-transfected MDCK cells, primary DRG cells and tumour cell lines were utilizedin vitro studies. OXA-induced chronic peripheral neurotoxicity mice and tumour-bearing nude mice were used in vivo studies. 


\section{Key Results}

OCT2 and OCTN1 but not OCTN2 were involved in the uptake of OXA; OCT2 played the most important role. L-THP reduced the cytotoxicity and cellular accumulation of OXA in a concentration-dependent manner in MDCK-hOCT2, MDCK-hOCTN1, and rat primary DRG cells but did not affect its efflux from MDCK-hMRP2 cells. Furthermore, L-THP attenuated OXA-induced peripheral neurotoxicity and reduced the platinum concentration in the DRG in mice in a dose-dependent manner. L-THP did not reduce the platinum concentration in the tumours and did not impair the antitumour efficacy of OXA in HT29 tumourbearing nude mice nor in tumour cells (HT29 and SW620 cells).

\section{Conclusion and Implications}

OCT2 and OCTN1, especially OCT2, contribute to OXA uptake in the DRG. L-THP attenuates OXAinduced peripheral neurotoxicity viainhibiting OCT2 and OCTN1 but without impairing the antitumour efficacy of OXA. L-THP is a potential candidate drug to attenuate OXA-induced peripheral neurotoxicity.

\section{Bullet point summary:}

What is already known:

Transporters play key roles in the accumulation of OXA in the DRG.

What this study adds:

1. L-THP attenuates OXA-induced peripheral neurotoxicity mainly by inhibiting OCT2.

2. L-THP does not impair the antitumor efficiency of OXA.

Clinical significance

1. The results help us to find a safe and efficient candidate to attenuate OXA-induced peripheral neurotoxicity.

2. The results reveal a new clinical use of L-THP.

\section{Abbreviations}

DRG, dorsal root ganglion; DMEM, Dulbecco's modified Eagle medium; FBS, Foetal bovine serum; HBSS, Hank's balanced salt solution; LDH, lactate dehydrogenase; L-THP, levo-tetrahydropalmatine; MATE, multidrug and toxin extrusion; MDCK, Madin-Darby canine kidney; MRP, multidrug resistance-associated protein; OCT, organic cation transporter; OCTN, organic cation/carnitine transporter; OXA, oxaliplatin; PBS, phosphate buffer saline; RMPI, Roswell Park Memorial Institute 1640 medium; SDS, sodium dodecyl sulfate; SEM, standard error

\section{Introduction}

Oxaliplatin (OXA), which belongs to the third generation of platinum antitumour drugs, is used for the treatment of multiple solid tumours, such as colorectal cancer and ovarian cancer (Staff \& Grisold et al., 2017). Compared to the first and second generation of platinum drugs, such as cisplatin and carboplatin, it shows the mildest nephrotoxicity and almost no ototoxicity clinically (Ruggiero \& Trombatore et al., 2013). However, OXA-induced severe and high-incidence neurotoxicity, especially peripheral neurotoxicity, has greatly limited its clinical application (Bano \& Najam et al., 2016).

It is reported that approximately $90 \%$ patients suffer from varying degrees of peripheral neurotoxicity, presented as muscle cramps, paraesthesia, and dysphasia, after the first administration of OXA (Argyriou \& Cavaletti et al., 2013). When the cumulative dose of OXA exceeds $1170 \mathrm{mg} / \mathrm{m}^{2}$, approximately $40-50 \%$ patients suffer from chronic peripheral neurotoxicity, which lasts for several months or even years after therapy (Argyriou \& Zolota et al., 2010). Therefore, OXA-induced peripheral neurotoxicity greatly limits its clinical application and affects the quality of life of patients. Because the mechanism underlying peripheral neurotoxicity is complex and unclear, reduced glutathione, a calcium-magnesium mixture, and a sodium ion 
channel blocker (Scuteri \& Galimberti A et al., 2010; Descoeur \& Pereira et al., 2011; Nieto \& Entrena et al., 2008) were recently evaluated for the clinical alleviation of peripheral neurotoxicity; however, these interventions did not show sufficient efficiency. Considering the wide clinical use of OXA, it is critical to explore new strategies for OXA-induced peripheral neurotoxicity.

OXA primarily damages the sensory neurons of the spinal dorsal root ganglion (DRG) (Avan \& Postma et al., 2015). Because DRG lacks the protection provided by the blood-brain barrier, it is particularly vulnerable to neurotoxic damage, explaining the occurrence of primarily sensory symptoms in chemotherapy-induced peripheral neurotoxicity (Carozzi \& Canta et al., 2015). It was found that the concentration of platinum in the DRG was much higher than that in the plasma, which indicated that platinum can be accumulated in the DRG (Screnci \& McKeage et al., 2000). Due to the poor passive permeability of OXA (Hall \& Okabe et al., 2008), membrane transporters probably play crucial roles in the transcellular transport of OXA (Johnson \& Jun et al., 2012). Therefore, the co-administration of a selective inhibitor of the transporter-mediated influx of OXA into DRG cells is probably an efficient strategy to attenuate OXA-induced peripheral neurotoxicity.

DRG cells express multiple ATP-binding transporters and solute carrier transporters (SLC). It has been reported that organic cation transporter 2 (OCT2) (Sprowl \& Ciarimboli et al., 2013; Cavaletti G, 2014; Burger \& Zoumaro et al., 2010), copper ion transporter 1 (CTR1) (Larson \& Blair et al., 2009), and carnitine/organic cation transporter 1 (OCTN1) (Jong \& Nakanishi et al., 2011) mediate OXA uptake in DRG cells, while MRP2 plays a crucial role in the efflux of OXA from DRG cells (Myint \& Li et al., 2015). Because CTR1 is also expressed in most tumours, such as human malignant tissues of colon carcinomas (Holzer \& Varki et al., 2006), and the expression of OCT2 in most tumour cells is down-regulated or even lost due to epigenetic modification (Ciarimboli G, 2014), therefore, the inhibition of OCT2 and OCTN1 but not CTR1 and MRP2 may attenuate OXA-induced peripheral neurotoxicity without influencing its antitumour effect. Our previous research showed that L-THP could strongly inhibit the activity of OCT2; the present study aims to explore whether L-THP would selectively inhibit OCT2 and OCTNs and subsequently alleviate OXA-induced peripheral neurotoxicity without impairing its antitumour effect.

\section{Methods}

\section{Chemicals and reagents}

Foetal bovine serum (FBS, No. 10099141), trypsin (No. 27250018), Dulbecco's modified Eagle's medium (DMEM, No. THP5218), L-Glutamine (No. 1816801), B-27 (No. 2004470), Neurobasal-A Medium (No. 1995169), and Roswell Park Memorial Institute 1640 (GI 1640) medium (No. 8119040) were purchased from Gibco Invitrogen Corporation (Carlsbad, CA, USA). Collagenase I (No. C0130-1G) and collagen (type I solution from rat tail, No. A10483-01) were obtained from Sigma-Aldrich (St. Louis, MO, USA). L-THP and OXA (No. D109812) were purchased from Aladdin Reagent Co., Ltd. (Shanghai, China).

\section{Cell culture}

MDCK cells stably transfected with plasmid vector pcDNA3.1(+) containing human OCT2 cDNA (MDCKhOCTN1) (Bai \& Ma et al., 2017), plasmid vector pcDNA3.1(+) containing human OCTN1 cDNA (MDCKhOCT2) (Li \& Weng et al., 2017), plasmid vector pcDNA3.1(+) containing human MRP2 cDNA (MDCKhOCTN2) (Ma \& Yang et al., 2017), and blank vector (mock cells) were all established in our laboratory and grown in DMEM medium supplemented with 10\% FBS and 1\% penicillin/streptomycin. SW620 and HT29 cell lines were obtained from the American Type Culture Collection (ATCC; Manassas, VA, USA) and grown in GI 1640 medium supplemented with 10\% FBS and 1\% penicillin/streptomycin. All cells were maintained in a humidified air $/ \mathrm{CO}_{2}$ incubator $(5 \% \mathrm{v} / \mathrm{v})$ at $37{ }^{\circ} \mathrm{C}$.

\section{Preparation of primary rat DRG cells}

DRG cells were isolated according to the methods described byBurkey et al. (Burkey \& Hingtgen et al., 2004), with some modification. Healthy Sprague-Dawley rats (male, $\sim 250 \mathrm{~g}$ ) were placed on the operating table after ether anaesthesia and disinfected with $75 \%$ ethanol. The spinal canals were separated, and the DRGs were collected in cold Hank's balanced salt solution (HBSS). All operations were performed quickly 
on ice. After digestion with $1.25 \mathrm{mg} / \mathrm{mL}$ collagenase $\mathrm{IV}\left(90 \mathrm{~min}, 37{ }^{\circ} \mathrm{C}\right)$ and $0.025 \% \operatorname{trypsin}\left(90 \mathrm{~min}, 37^{\circ} \mathrm{C}\right)$, the DRG cells were dispersed in a medium containing $50 \mu \mathrm{M} 5$-fluro-2'-deoxyuridine. Sensory neurons were plated at 28,000-30,000 cells per well in 6-well tissue-culture plates or 7500-8000 cells per well in 96-well tissue-culture plates. The cells were maintained at $37^{\circ} \mathrm{C}$ under a $5 \% \mathrm{CO} 2$ atmosphere.

\section{Cytotoxicity study}

MDCK-hOCTN1, MDCK-hOCTN2, MDCK-hOCT2, MDCK-hMRP2 and mock cells in the logarithmic growth phase were digested and collected. The cells were resuspended in fresh medium, adjusted to a cell density of $4.0 \times 10^{4} / \mathrm{mL}$, and seeded in 96 -well cell plates at $0.2 \mathrm{~mL} /$ well. After the above cells and primary DRG cells were cultured for $24 \mathrm{~h}$, the cells were treated with $40 \mu \mathrm{M}$ OXA in the absence or presence of L-THP for another $48 \mathrm{~h}$, a part of the medium was collected for the measurement of lactate dehydrogenase (LDH) activity using the LDH assay kit, and the cell viability was evaluated by the MTT assay (Tu \& Li et al., 2014; Li \& Song et al., 2016).

\section{Cellular uptake study}

The cellular accumulation of OXA was evaluated according to a previously reported method with minor modifications (Ma \& Yang et al., 2017). The cells were seeded in 12-well plates (for mock and transporter transfected cells) or 6-well plates (for DRG cells) (Corning Costar, NY, USA) and cultured to $90 \%$ confluence. MDCK-hOCTN1, MDCK-hOCT2, MDCK-hOCTN2, mock, and primary DRG cells were preincubated with HBSS at $37{ }^{\circ} \mathrm{C}$ for $5 \mathrm{~min}$, while MDCK-hMRP2 cells were incubated for $30 \mathrm{~min}$. Next, 0.5 $\mathrm{mL}$ of HBSS containing $40 \mu \mathrm{M}$ OXA with or without L-THP was added to initiate cellular uptake. The experiment was performed in a humidified air $/ \mathrm{CO}_{2}$ incubator $\left(5 \% \mathrm{v} / \mathrm{v}\right.$ ) at $37{ }^{\circ} \mathrm{C}$ for $5 \mathrm{~min}$ (for uptake transporters) or $1 \mathrm{~h}$ (for efflux transporter MRP2) and terminated by removing the incubation buffer rapidly. Next, the cells were quickly rinsed three times with ice-cold HBSS and lysed using $100 \mu \mathrm{L}$ of $0.01 \%$ SDS.

\section{Animal treatment}

All animal procedures and study protocols were double-blind and carried out in strict accordance with the recommendations of the Guide for the Institutional Animal Care and Use Committee of Zhejiang University Medical Center. Mice were housed in cages at a controlled temperature $\left(22 \pm 0.1{ }^{\circ} \mathrm{C}\right)$ and humidity $(50$ $\pm 10 \%)$ with a 12-h light/dark cycle and provided with free access to food and water. All surgeries were performed under chloral hydrate anaesthesia, and all efforts were made to minimize animal suffering. All mice were killed by $\mathrm{CO}_{2}$ asphyxiation. Animals were randomised for treatment.

Specific pathogen-free ICR mice (22-25 g), aged 4 weeks (male, 8; female, 8), were purchased from the Experimental Animal Center of the Zhejiang Academy of Medical Sciences [SCXK (Zhe) 2014-0001]. Nude mice (5 weeks old, male) were obtained from Beijing Vital River Laboratory Animal Technology Company (Beijing, China; SCXK [Jing] 2012-0001) and maintained under specific pathogen-free (SPF) conditions. After adaptive feeding for one week, the nude mice were subcutaneously injected with HT29 cells into the rear flanks $\left(1 \times 10^{7}\right.$ cells $/$ side $)$. Nude mice with tumours of average volume $=50-60 \mathrm{~mm}^{3}$ were selected for the experiments.

To evaluate the neuroprotective effects of L-THP, mice with one-week adaptive feeding, were randomly allocated into 6 groups with 16 mice each (half male and half female): vehicle group, OXA-alone group, OXA and L-THP co-treatment groups, and L-THP-alone group. OXA was intravenously injected ( $i v, 8$ $\mathrm{mg} / \mathrm{kg}$ ) twice a week for a total of four weeks (days 1, 4, 8, 11, 15, 18, 22, and 25). For the co-treatment groups, the mice were $i v$ injected with $5 \%$ glucose solution containing L-THP $(5-20 \mathrm{mg} / \mathrm{kg})$ and OXA (8 $\mathrm{mg} / \mathrm{kg}$ ). Mice in the vehicle or L-THP-alone groups received equal volumes of $5 \%$ glucose without or with L-THP $(10 \mathrm{mg} / \mathrm{kg})$, respectively. The mouse behaviour and body weight were monitored and recorded after every administration. Twenty-four hours after the final administration, the animals were sacrificed and the DRGs were collected for analysis.

For evaluation of the antitumour activity, the tumour-bearing nude mice were allocated into 4 groups to evaluate whether L-THP co-treatment (10 and $20 \mathrm{mg} / \mathrm{kg}$ ) would impair the antitumour efficacy of OXA 
( $8 \mathrm{mg} / \mathrm{kg}$ ): vehicle group, OXA-alone group, and OXA and L-THP co-treatment groups. Drugs were administered intravenously every two days for two weeks (days $1,3,5,7,9,11$, and 13), and the body weight and tumour size were monitored. Twenty-four hours after the final administration, the animals were sacrificed, and the tumours were collected, weighted, and stored for analysis. The tumour sizes were calculated according to the following formula: tumour size $=a^{*} \boldsymbol{b}^{\mathbf{2}} / \mathbf{2}$, where a is the long side of the tumour and $\mathrm{b}$ is the short side of the tumour.

\section{Behavioural tests}

All behavioural tests were performed $24 \mathrm{~h}$ after administration (days 2, 5, 9, 12, 16, 19, 23, and 26). The thermal nociceptive threshold was assessed using the ZH-6C cold-plate (Anhui Zhenghua Biological Instrument Equipment Co., Ltd.). The mice were placed on the cold-plate floor. The temperature of the cold plate was kept constant at 4.0-4.2 degC. After allowing the mice to adapt for $1 \mathrm{~min}$, the frequency of hind-paw licking by the mice was recorded for 2 min.

\section{Determination of OXA concentration by ICP-MS}

We added $200 \mu \mathrm{L}$ of $\mathrm{HNO}_{3}$ and $400 \mu \mathrm{L}$ of $\mathrm{H}_{2} \mathrm{O}_{2}$ into the lysate of the collected cells, DRGs, or tumours; the mixture was incubated for $2 \mathrm{~h}$ at 80 . Next, $200 \mu \mathrm{L}$ of $25 \%$ ammonia solution was added, and the volume of the solution was adjusted to $4.0 \mathrm{~mL}$ with $\mathrm{ddH}_{2} 0$. Finally, the concentrations of OXA were determined by inductively coupled plasma mass spectrometry (ICP-MS, PerkinElmer Nexion 300X) after the solution was filtered using a film filter $(0.22 \mu \mathrm{m})$. The OXA content was determined relative to the total protein content for cells or relative to the weight for tissues.

\section{Statistical analysis}

All experiments and data analysis were blinded. The median lethal dose $\left(\mathrm{LD}_{50}\right)$ was obtained through sigmoid curve fitting by plotting cell viability versus the $\log 10$ OXA concentrations. Data are presented as the means \pm standard error of the mean (SEM). For thein vitro data, each point was obtained from at least two independent experiments with five or six samples each. Unpaired two-tailed Student's $t$ test was used to analyse differences between two groups, and one-way analysis of variance (ANOVA) followed by Bonferroni's or Dunnett's post hoctest was used for multiple-group comparisons. A $P$-value $<0.05$ was considered statistically significant. All data analyses were performed using GraphPad Prism 5.0 (GraphPad Software Inc., San Diego, CA, USA).

\section{Results}

\section{OXA exhibited the highest toxicity and accumulation in MDCK-hOCT2 cells}

To confirm the roles of OCT2, OCTN1, and OCTN2 in the uptake of OXA, we compared OXA-induced toxicity in transporter-transfected MDCK cells. Our results revealed that the order of sensitivity to OXA was MDCK-hOCT2 (LD $\left.{ }_{50}: 3.3 \pm 0.7 \mu \mathrm{M}\right)$ i MDCK-hOCTN1 $\left(\mathrm{LD}_{50}: 32.5 \pm 1.1 \mu \mathrm{M}\right)$ i MDCK-hOCTN2 [?] mock cells $\left(\mathrm{LD}_{50} i, 100 \mu \mathrm{M}\right)$ (Figure 1a ). We further showed that the cellular accumulation of OXA in the MDCK-hOCT2 and MDCK-hOCTN1 cells were 5.4 and 2.0 folds that in the mock cells (Figure 1b ), respectively. The above results imply that OCT2 and OCTN1 but not OCTN2 contribute to the uptake of OXA, and OCT2 plays the most crucial role.

\section{L-THP attenuated OXA-induced toxicity and reduced the accumulation of OXA in OCT2- and OCTN1-transfected cells}

Our previous study demonstrated that L-THP was a strong inhibitor of OCT2, we studied the inhibitory effect of L-THP on OXA-induced toxicity in mock and MDCK-hOCTN1 cells, as well as MDCK-hOCT2 cells. As we described above, $40 \mu \mathrm{M}$ of OXA reduced the cell viability to $76 \%, 50 \%$, and $12 \%$ of the control (vehicle treatment) in the mock, MDCK-hOCTN1, and MDCK-hOCT2 cells, respectively, while the LDH activities in the medium were increased to 1.1, 1.7, and 4.0 folds, respectively. In the MDCK-hOCTN1 and MDCK-hOCT2 cells, 1-100 $\mu \mathrm{M}$ of L-THP attenuated OXA-induced toxicity in a concentration-dependent manner, with increase in cell viability and reduction of LDH leakage in the cell medium; particularly, in the 
MCDK-OCT2 cells, the cell viability was increased to $64 \%$ (vs 12\%) and the LDH was reduced to half of that in the OXA group (Figure 2a-2f ).

On the other hand, the cellular accumulation of OXA ( $40 \mu \mathrm{M}, 30$ min incubation) in the MDCK-hOCTN1 and MDCK-hOCT2 cells was approximately 2.0 and 5.4 folds that in mock cells, respectively, whereas coincubation with $100 \mu \mathrm{M}$ L-THP reduced the OXA accumulation in the MDCK-hOCTN1 and MDCK-hOCT2 cells to 1.1 and 2.8 folds that in the mock cells (Figure $\mathbf{3}$ ). The results indicate that L-THP significantly inhibits OCT2- and OCTN1-mediated OXA uptake, exhibiting a considerably stronger inhibition of hOCT2 than hOCTN1, which implies that L-THP can significantly inhibit OCT2- and OCTN1-mediated OXA uptake and subsequently attenuate OXA-induced toxicity.

\section{L-THP did not inhibit cellular accumulation of OXA in MDCK-hMRP2}

Since MRP2 is the most important transporter mediating the efflux of OXA from DRG cells, we evaluated the effect of L-THP on MRP2 to elucidate whether L-THP would reduce OXA efflux by inhibiting MRP2. As shown inFigure 4, the accumulation of OXA in MDCK-MRP2 cells was approximately half of that in the mock cells, and 1-100 $\mu \mathrm{M}$ L-THP did not increase the accumulation, which indicates that L-THP does not decrease OXA efflux by inhibition of MRP2.

\section{L-THP reduced OXA accumulation and attenuated OXA-induced toxicity in primary DRG cells}

Since OCT2 and MRP2 play vital roles in the uptake and efflux of OXA, respectively, and L-THP strongly inhibited OCT2 activity but not MRP2, we deduced that L-THP could reduce OXA accumulation and attenuate OXA-induced toxicity in DRG. Our results revealed that $40 \mu \mathrm{M}$ of OXA significantly reduced the cell viability of primary DRG cells (to approximately $52 \%$ of the control), while co-treatment with L-THP $(1-100 \mu \mathrm{M})$ increased the cell viability in a concentration-dependent manner (Figure 5a) ; for instance, 10 $\mu \mathrm{M}$ of L-THP increased the cell viability to $75 \%$ of the control, which indicates that L-THP may reduce the toxicity of OXA in DRG cells.

In agreement with the result of the cell viability test, L-THP $(1-100 \mu \mathrm{M})$ reduced the accumulation of OXA in primary DRG cells in a concentration-dependent manner; for example, L-THP at $100 \mu \mathrm{M}$ reduced the platinum accumulation in primary DRG cells to $32 \%$ of that in the OXA-alone group (Figure $\mathbf{5 b}$ ). In summary, our results suggest that L-THP can decrease the cellular accumulation of OXA and can thereby ameliorate OXA-induced toxicity in primary DRG cells.

\section{L-THP ameliorated OXA-induced neurotoxicity and reduced platinum accumulation in DRG of mice}

To further confirm whether L-THP could attenuate OXA-induced neurotoxicity in vivo, we performed a behavioural test and detected platinum accumulation in DRG in OXA-treated mice without or with LTHP co-treatment. As shown in Figure 6, $24 \mathrm{~h}$ after the final administration, the sensitivity to cold stimulation, expressed as the frequency of hind-paw licking in the mice treated with OXA $(8 \mathrm{mg} / \mathrm{kg}$, iv $)$, was approximately 3 folds that in the vehicle-treated male or female mice. L-THP $\left(5^{\sim} 20 \mathrm{mg} / \mathrm{kg}\right)$ dose-dependently attenuated the increase in OXA-induced cold-stimulation sensitivity (Figure 6a and 6b ). Accordingly, the concentration of platinum in the DRG reached $2.35 \mathrm{ng} / \mathrm{mg}$ in mice treated with OXA $(8 \mathrm{mg} / \mathrm{kg})$, while LTHP reduced the concentration of platinum in the DRG in a dose-dependent $(5-20 \mathrm{mg} / \mathrm{kg}$ ) manner (Figure 6c ). The L-THP-alone group and the vehicle-alone group did not show a significant difference in coldstimulation sensitivity, indicating that the abirritation of L-THP could be excluded. Therefore, L-THP ameliorated OXA-induced peripheral neurotoxicity by inhibiting the uptake of OXA in DRG cells rather than by abirritation.

L-THP did not impair the antitumour efficacy of OXA in tumour cells nor in tumour-bearing nude mice

To explore whether L-THP might impair the antitumour efficiency of OXA, the cytotoxicity and antitumour 
effect of OXA were evaluated in HT29 and SW620 cells, as well as in HT29 tumour-bearing nude mice with or without L-THP. As shown in Figures 7a and 7b, co-incubation with L-THP (1-100 $\mu \mathrm{M})$ did not reduce the cytotoxicity of OXA in the HT29 or SW620 cells. In agreement with the results in vitro, the tumour growth was markedly inhibited in the OXA-alone $(8 \mathrm{mg} / \mathrm{kg}$, single dose) group, as well as in the L-THP co-treated (10 and $20 \mathrm{mg} / \mathrm{kg}$ ) groups. Meanwhile, the concentration of OXA in tumours showed no significant difference between the OXA-alone or L-THP co-administration groups.

\section{Discussion}

OXA is an effective drug for the treatment of solid tumours such as colorectal cancer; however, OXAinduced peripheral neurotoxicity reduces the life quality of patients and greatly limits the use of OXA in the clinic. The present study confirmed that OCT2 and OCTN1, especially OCT2, play vital roles in the uptake of OXA, and MRP2 is involved in the efflux of OXA. The further study demonstrated that L-THP could inhibit OCT2 and OCTN1 activities but not MRP2, reduce the accumulation of OXA in the DRG, and subsequently attenuate OXA-induced peripheral neurotoxicity. Finally, our results demonstrated that L-THP did not reduce the accumulation of OXA in tumours; therefore, it did not impair the antitumour efficiency of OXA.

Although several published papers have shown that OCT2, OCTN1, and CTR1 contribute to the uptake of OXA, to further compare the role of individual transporters in the uptake of OXA, we first estimated the OXA-induced cell toxicity and OXA accumulation in mock, MDCK-hOCTN1, MDCK-hOCTN2, and MDCK-hOCT2 cells. Our data demonstrated that OXA is a substrate of OCTN1 and OCT2 but not that of OCTN2. Furthermore, OCT2 played a more important role in the uptake of OXA because the $\mathrm{LD}_{50}$ of OXA in MDCK-hOCT2 cells $(3.4 \mu \mathrm{M})$ was much lower than that in MDCK-hOCTN1 $(25.8 \mu \mathrm{M})$, and the cellular accumulation of OXA was much higher in MDCK-hOCT2 cells than that in MDCK-hOCTN1 cells (5.4 vs 2.0 folds that of the mock cells).

Because we did not have a CTR1-overexpression cell model, we did not study the role of CTR1 in the uptake of OXA. However, as reported by Burger et al., the inhibitor of CTR1, copper histidine (Ip \& Liu et al., 2013), did not exhibit a significant influence on OXA uptake in DRG cells, which indicates that the role of CTR1 in OXA uptake may not be essential in DRG. Considering that CTR1 is also expressed in human malignant tissues, such as colon carcinomas, it is not a good target for the inhibition of OXA-induced peripheral neurotoxicity. Our study result demonstrated that the concentration of platinum in the tumour of the nude mice co-treated with L-THP and OXA was not obviously altered, when compared with that in the nude mice treated with OXA alone, which implies that L-THP did not inhibit CTR1; therefore, it is reasonable that L-THP did not impair the antitumour efficiency of OXA.

Additionally, ATP7A/7B (Ip \& Liu et al., 2010; Li \& Yin et al., 2018; Lasorsa \& Nardella et al., 2019), MRP2 (Myint \& Li et al., 2015; Myint \& Biswas et al., 2019), and MATE1 (Nies \& Koepsell et al., 2011; Fujita \& Hirota et al., 2019) are involved in OXA efflux in the DRG, and MRP2 was reported as one of the most important transporters in the efflux of OXA from DRG cells. Our result showed that the OXA accumulation in MDCK-MRP2 cells was much lower than that in mock cells, and $100 \mu \mathrm{M}$ L-THP did not obviously influence the accumulation of OXA in MDCK-MRP2 cells and mock cells, indicating that L-THP does not influence OXA accumulation in the DRG and tumour tissues via inhibiting MRP2. Moreover, our previous study revealed that L-THP did not affect the function of MATE1 (Li \& Song et al., 2016). Since we did not have an ATP7A/7B-transfected cell model, ATP7A/7B was not considered in the present study. Based on the result that L-THP significantly decreased OXA accumulation in the DRG in vitro and in vivo , we speculate that L-THP mainly inhibits OXA uptake in the DRG rather than its efflux.

Although OCT2 plays the most crucial role in OXA uptake in the DRG, it is extremely down-regulated or even lost in most tumours due to epigenetic modification (Zhu \& Yu et al., 2019; Chen \& Qin et al., 2019). Therefore, selective inhibition of OCT2 would not impair the antitumour effect of OXA. As expected, our present results showed that co-incubation with L-THP did not decrease the cell toxicity of OXA in colorectal cancer cell lines (the main clinical indication of OXA). Thein vivo results further revealed that 
co-administration of L-THP did not reduce the tumour accumulation of OXA and impair the antitumour efficacy of OXA.

L-THP has been used in China as an analgesic with sedative/hypnotic properties for more than 40 years (Liu \& Wang et al., 2019; Gong \& Yue et al., 2016; Du \& Du et al., 2017). OXA-induced peripheral neurotoxicity increases cold-stimulation sensitivity, as indicated by an increase in the frequency of lapping hind paws. To exclude the sedative/hypnotic effect of L-THP, we included a group that was treated with L-THP alone. As we observed, twenty-four hours after the L-THP administration, the mice did not exhibit sedative or hypnotic effects, and further behaviour evaluation showed no significant change between the vehicle control group and the group administered L-THP alone. The above observation implied that the reduction of cold-stimulation sensitivity in the group that was co-administered L-THP was irrelevant to its sedative/hypnotic effect. The significant reduction of OXA in the DRG of the mice by L-THP directly proved that the inhibitory effect of L-THP on OCT2 may be the important factor in the inhibition of OXA-induced peripheral neurotoxicity.

To study whether OXA-induced peripheral neurotoxicity exhibits gender difference, 16 mice were allocated in one group (half male and half female) in the behavioural test. To meet the requirements of the detection limit of platinum, the DRGs from two mice of the same group were pooled as one sample. The behavioural test (Figure $5 \mathrm{a}$ and $5 \mathrm{~b}$ ) showed that OXA-induced peripheral neurotoxicity showed no gender difference; therefore, the platinum concentration in the DRGs of male and female mice from the same group was combined. Originally, we had aimed to evaluate the antitumour effect of OXA in tumour-bearing nude mice for 3 weeks; however, the tumours in the vehicle group grew so fast that we had to terminate the experiment at the end of two weeks. Fortunately, we can conclude that L-THP did not impair the antitumour efficiency of OXA, based on the results from comparing the platinum concentration in the tumours and the tumour size between the OXA-alone group and L-THP co-treatment groups.

Considering that OXA can induce mitochondrial dysfunction (Cheng \& Xiang et al., 2019; Trecarichi \& Flatters, 2019; Toyama \& Shimoyama et al., 2018), it is interesting and meaningful to identify how OXA enters the mitochondria because of its poor passive permeability. OCTN1 has been reported to be localized in the mammalian mitochondrial membrane (Lamhonwah \& Tein, 2006), and our western blotting analysis also showed that OCT2 and OCTN1 were expressed in the mitochondria of rat DRGs (data not shown). Considering that L-THP passes easily across the cell membrane due to its high passive permeability, we speculated that L-THP probably inhibited OXA transport across the mitochondrial membrane. Apart from the SLC22A family, the SLC25A family has also been determined to play an important role in the mitochondrial carrier system (Taylor, 2017), which deserves consideration.

In summary, the present study confirmed the crucial role of OCT2 and OCTN1 but not OCTN2 in the uptake of OXA in the DRG and demonstrated that L-THP could prevent OXA-induced peripheral neurotoxicity through inhibiting OCT2 and OCTN1, without impairing the antitumour effect of OXA. Considering the safety of L-THP in clinical studies, L-THP is likely a potential candidate to attenuate OXA-induced peripheral neurotoxicity.

Authorship Contributions

Y. D. Y., L. P. L. and H. D. J. designed the research. Y. D. Y., L. P. L., F. F. S., P. L. M. Y. C., Y. C. C. and S. X. N. performed the research. Y. D. Y., L. P. L., M. Y. C. Y. C. C. and H. Z., S. Z. analyzed data. Y. D. Y., M. Y. C. and H. D. J. wrote the paper.

\section{References}

Staff NP, Grisold A, Grisold W, Windebank AJ. (2017). Chemotherapy-Induced Peripheral Neuropathy: A Current Review [J]. Ann Neurol, 81(6): 772-781.

Ruggiero A, Trombatore G, Triarico S, Arena R, Ferrara P, Scalzone M, .. Riccardi R. (2013). Platinum compounds in children with cancer: toxicity and clinical management. Anti-Cancer Drugs [J]. Anticancer Drugs, 24: 1007-1019. 
Bano N, Najam R, Qazi F, Mateen A. (2016). Clinical Features of OXA Induced Hypersensitivity Reactions and Therapeutic Approaches [J]. Asian Pac J Cancer Prev, 17(4): 1637-1641.

Argyriou AA, Cavaletti G, Antonacopoulou A, Genazzani AA, Briani C, Bruna J, .. Kalofonos H. (2013). Voltage-gated sodium channel polymorphisms play a pivotal role in the development of OXA-induced peripheral neurotoxicity: results from a prospective multicenter study [J]. Cancer, 119: 3570-3577.

Argyriou AA, Zolota V, Kyriakopoulou O, Kalofonos HP. (2010). Toxic peripheral neuropathy associated with commonly used chemotherapeutic agents [J]. J BUON, 15: 435-446.

Scuteri A, Galimberti A, Ravasi M, Pasini S, Donzelli E, Cavaletti G, Tredici G. (2010). NGF protects dorsal root ganglion neurons from OXA by modulating JNK/Sapk and ERK1/2 [J]. Neurosci Lett, 486(3): 141-145.

Descoeur J, Pereira V, Pizzoccaro A, Francois A, Ling B, Maffre V, ... Bourinet E. (2011). OXA-induced cold hypersensitivity is due to remodelling of ion channel expression in nociceptors $[\mathrm{J}]$. EMBO Mol Med, 3(5): 266-278.

Nieto FR, Entrena JM, Cendán CM, Pozo ED, Vela JM, Baeyens JM. (2008). Tetrodotoxin inhibits the development and expression of neuropathic pain induced by paclitaxel in mice [J]. Pain, 137(3): 520-531.

Avan A, Postma TJ, Ceresa C, Avan A, Cavaletti G, Giovannetti E, Peters GJ. (2015). Platinum-induced neurotoxicity and preventive strategies: past, present, and future [J]. Oncologist, 20(4): 411-432.

Carozzi VA, Canta A, Chiorazzi A. (2015). Chemotherapy-induced peripheral neuropathy: What do we know about mechanisms? [J]. Neurosci Lett, 596: 90-107.

Screnci D, McKeage MJ, Galettis P, Hambley TW, Palmer BD, Baguley BC. (2000). Relationships between hydrophobicity, reactivity, accumulation and peripheral nerve toxicity of a series of platinum drugs $[\mathrm{J}]$. Br. J. Cancer, 82: 966-972.

Hall MD, Okabe M, Shen DW, Liang XJ, Gottesman MM. (2008). The role of cellular accumulation in determining sensitivity to platinum-based chemotherapy [J]. Annu Rev Pharmacol Toxicol, 48: 495-535.

Johnson JL, Jun L and Mark JM. (2012). Membrane Transporters as Determinants of the Pharmacology of Platinum Anticancer Drugs [J]. Current Cancer Drug Targets, 12: 962-986.

Sprowl JA, Ciarimboli G, Lancaster CS, Giovinazzo H, Gibson AA, Du G, ... Sparreboom A. (2013). OXA induced neurotoxicity is dependent on the organic cation transporter OCT2 [J]. Proc Natl Acad Sci USA, 110: 11199-11204.

Cavaletti G. (2014). Chemotherapy-induced peripheral neurotoxicity (CIPN): what we need and what we know [J]. J Peripher Nerv Syst, 19(2): 66-76.

Burger H, Zoumaro DA, Boersma AW, Helleman J, Berns EM, Mathijssen RH, ... Wiemer EA. (2010). Differential transport of platinum compounds by the human organic cation transporter hOCT2 (hSLC22A2) [J]. Br J Pharmacol, 159(4): 898-908.

Larson CA, Blair BG, Safaei R, Howell SB. (2009). The role of the mammalian copper transporter 1 in the cellular accumulation of platinum-based drugs [J]. Mol Pharmacol, 75(2): 324-330.

Jong NN, Nakanishi T, Liu JJ, Tamai I, McKeage MJ. (2011). OXA transport mediated by organic cation/carnitine transporters OCTN1 and OCTN2 in overexpressing human embryonic kidney 293 cells and rat dorsal root ganglion neurons [J]. J Pharmacol Exp Ther, 338(2): 537-547.

Myint K, Li Y, Paxton J, McKeage M. (2015). Multidrug Resistance-Associated Protein 2 (MRP2) Mediated Transport of OXA-Derived Platinum in Membrane Vesicles [J]. PLoS One, 1(10): 7.

Holzer AK, Varki NM, Le QT, Gibson, MA, Naredi P, Howell SB. (2006). Expression of the human copper influx transporter 1 in normal and malignant human tissues [J]. J Histochem Cytochem, 54: 1041-1049. 
Ciarimboli G. (2014). Membrane transporters as mediators of cisplatin side-effects [J]. Anticancer Res, 34(1): 547-550.

Bai M, Ma Z, Sun D, Zheng C, Weng Y, Yang X, .. Jiang H. (2017). Multiple drug transporters mediate the placental transport of sulpiride [J]. Arch Toxicol, 91(12): 3873-3884.

Li L, Weng Y, Wang W, Bai M, Lei H, Zhou H, Jiang H. (2017). Multiple organic cation transporters contribute to the renal transport of sulpiride [J]. Biopharm Drug Dispos, 38(9): 526-534.

Ma Z, Yang X, Jiang T, Bai M, Zheng C, Zeng S, .. Jiang H. (2017). Multiple SLC and ABC Transporters Contribute to the Placental Transfer of Entecavir [J]. Drug Metab Dispos, 45(3): 269-278.

Burkey T, Hingtgen CM, Vasko MR. (2004). Isolation and culture of sensory neurons from the dorsal-root ganglia of embryonic or adult rats [J]. Methods Mol Med, 99: 189-202.

Tu M, Li L, Lei H, Ma Z, Chen Z, Sun S, ... Jiang H. (2014). Involvement of Organic Cation Transporter1 and Cytochrome P450 3A4 in Retrorsine Induced Toxicity [J]. Toxicology, 322: 34-42.

Li LP, Song FF, Weng YY, Yang X, Wang K, Lei HM, ... Jiang HD. (2016). Role of OCT2 and MATE1 in renal disposition and toxicity of nitidine chloride [J]. Br J Pharmacol, 173(16): 2543-2554.

Burkey TH, Hingtgen CM, Vasko MR. (2004). Isolation and culture of sensory neurons from the dorsal-root ganglia of embryonic or adult rats [J]. Methods Mol Med, 99: 189-202.

Ip V, Liu JJ, McKeage MJ. (2013). Evaluation of effects of copper histidine on copper transporter 1-mediated accumulation of platinum and oxaliplatin-induced neurotoxicity in vitro and in vivo $[\mathrm{J}]$. Clin Exp Pharmacol Physiol, 40(6): 371-378.

Ip V, Liu JJ, Mercer JF, McKeage MJ. (2010). Differential expression of ATP7A, ATP7B and CTR1 in adult rat dorsal root ganglion tissue [J]. Mol Pain, 13(6): 53.

Li YQ, Yin JY, Liu ZQ, Li XP. (2008). Copper efflux transporters ATP7A and ATP7B: Novel biomarkers for platinum drug resistance and targets for therapy [J]. IUBMB Life, 70(3): 183-191.

Lasorsa A, Nardella MI, Rosato A, Mirabelli V, Caliandro R, Caliandro R, ... Arnesano F. (2019). Mechanistic and Structural Basis for Inhibition of Copper Trafficking by Platinum Anticancer Drugs [J]. J Am Chem Soc, 141(30): 12109-12120.

Myint K, Li Y, Paxton J, McKeage M. (2015). Multidrug Resistance-Associated Protein 2 (MRP2) Mediated Transport of OXA-Derived Platinum in Membrane Vesicles [J]. PLoS One, 1(10): 7.

Myint K, Biswas R, Li Y, Jong N, Jamieson S, Liu J, .. McKeage M. (2019). Identification of MRP2 as a targetable factor limiting OXA accumulation and response in gastrointestinal cancer [J]. Sci Rep, 9(1): 2245

Nies AT, Koepsell H, Damme K, Schwab M. (2011). Organic cation transporters (OCTs, MATEs), in vitro and in vivo evidence for the importance in drug therapy [J]. Handb Exp Pharmacol, (201): 105-167.

Fujita S, Hirota T, Sakiyama R, Baba M, Ieiri I. (2019). Identification of drug transporters contributing to OXA-induced peripheral neuropathy [J]. J Neurochem, 148(3): 373-385.

Zhu Q, Yu L, Qin Z, Chen L, Hu H, Zheng X, Zeng S. (2019). Regulation of OCT2 transcriptional repression by histone acetylation in renal cell carcinoma [J]. Epigenetics, 14(8): 791-803.

Chen L, Qin Z, Lei J, Ye S, Zeng K, Wang H, .. Yu L. (2019). Upregulation of miR-489-3p and miR-630 inhibits OXA uptake in renal cell carcinoma by targeting OCT2 [J]. Acta Pharm Sin B, 9(5): 1008-1020.

Liu YY, Wang TX, Zhou JC, Qu WM, Huang ZL. (2019). Dopamine D1 and D2 receptors mediate analgesic and hypnotic effects of l-tetrahydropalmatine in a mouse neuropathic pain model [J]. Psychopharmacology (Berl), 236(11): 3169-3182. 
Gong X, Yue K, Ma B, Xing J, Gan Y, Wang D, .. Li C. (2016). Levo-tetrahydropalmatine, a natural, mixed dopamine receptor antagonist, inhibits methamphetamine self-administration and methamphetamineinduced reinstatement [J]. Pharmacol Biochem Behav, 144: 67-72.

Du Y, Du L, Cao J, Holscher C, Feng Y, Su H, ... Yun KM. (2017). Levo-tetrahydropalmatine inhibits the acquisition of ketamine-induced conditioned place preference by regulating the expression of ERK and CREB phosphorylation in rats [J]. Behav Brain Res, 15(317): 367-373.

Cheng W, Xiang W, Wang S, Xu K. (2019). Tanshinone IIA ameliorates OXA-induced neurotoxicity via mitochondrial protection and autophagy promotion [J]. Am J Transl Res. 2019, 11(5): 3140-3149.

Trecarichi A, Flatters SJL. (2019). Mitochondrial dysfunction in the pathogenesis of chemotherapy-induced peripheral neuropathy $[\mathrm{J}]$. Int Rev Neurobiol, 145: 83-126.

Toyama S, Shimoyama N, Szeto HH, Schiller PW, Shimoyama M. (2018). Protective Effect of a MitochondriaTargeted Peptide against the Development of Chemotherapy-Induced Peripheral Neuropathy in Mice [J]. ACS Chem Neurosci, 9(7): 1566-1571.

Lamhonwah AM, Tein I. (2006). Novel localization of OCTN1, an organic cation/carnitine transporter, to mammalian mitochondria [J]. Biochem Biophys Res Commun, 345(4): 1315-1325.

Taylor EB. (2017). Functional Properties of the Mitochondrial Carrier System [J]. Trends Cell Biol, 27(9): 633-644.
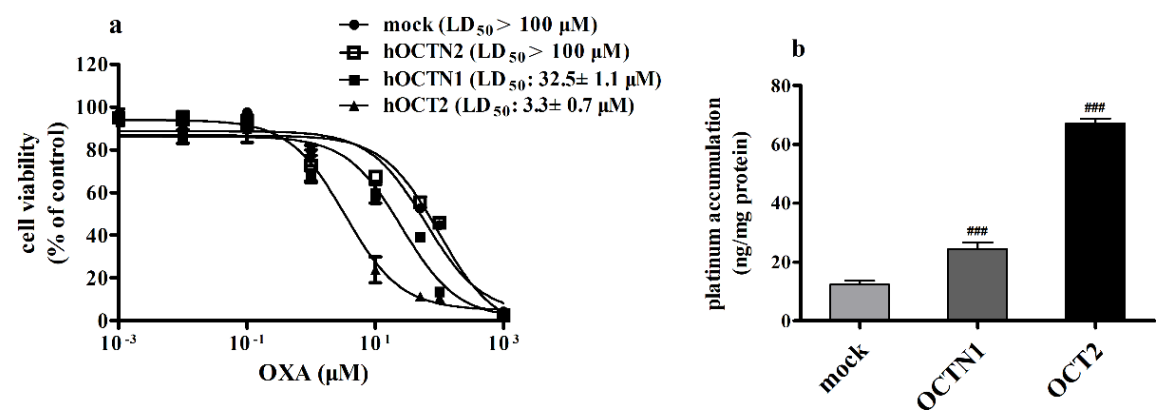

Figure 1. Concentration-dependent cytotoxicity (a) and cellular accumulation (b) of OXA in mock and hOCTN1-, hOCT2-, hOCTN2-transfected MDCK cells. For the cytotoxicity study, the cells were incubated in the absence or presence of OXA for $48 \mathrm{~h}$; for the cellular accumulation study, the cells were incubated with OXA $(40 \mu \mathrm{M})$ for 5 min. Data are expressed as the mean \pm SEM, $\mathrm{n}=6$ for cell toxicity and $\mathrm{n}=5$ for OXA accumulation. Compared with the mock cells, ${ }^{\#} P<0.01$, $\# \#+P<0.001$.
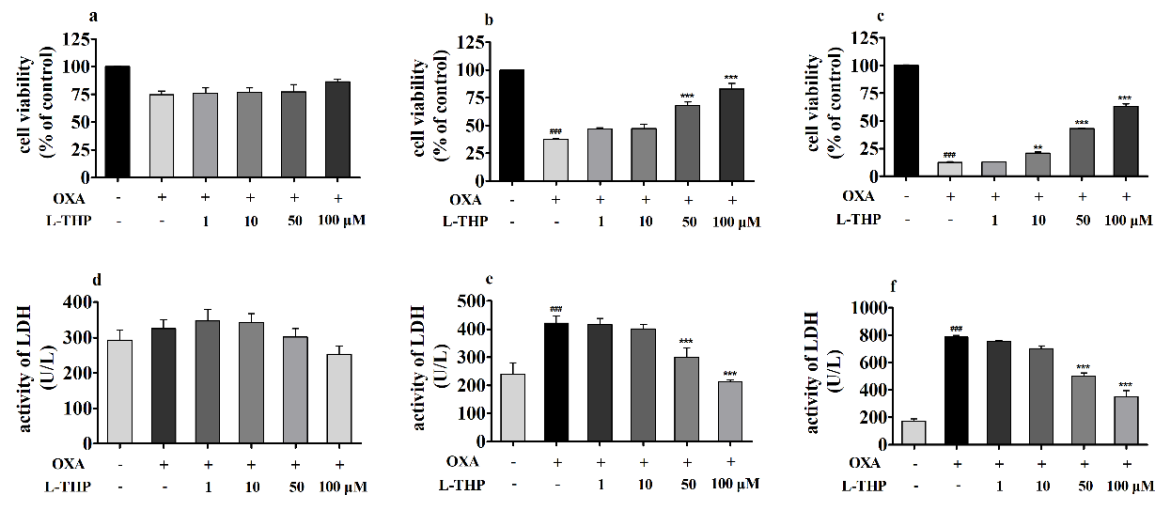
Figure 2. The effect of L-THP on OXA-induced cytotoxicity in mock, MDCK-hOCTN1, and MDCKhOCT2 cells. Cell viability of mock (a), MDCK-hOCTN1(b), and MDCK-hOCT2 (c) cells. LDH activities in the medium for mock (d), MDCK-hOCTN1 (e), and MDCK-hOCT2 (f) cells. In the toxicity study, the cells were incubated with or without OXA and/or L-THP for $48 \mathrm{~h}$. Data are expressed as the mean \pm $\mathrm{SEM}, \mathrm{n}=5$. Compared with the vehicle group, ${ }^{\# \# \#} P<0.001$; compared with the OXA-alone group, ${ }^{* *} P<$ 0.01 and $^{* * *} P<0.001$.

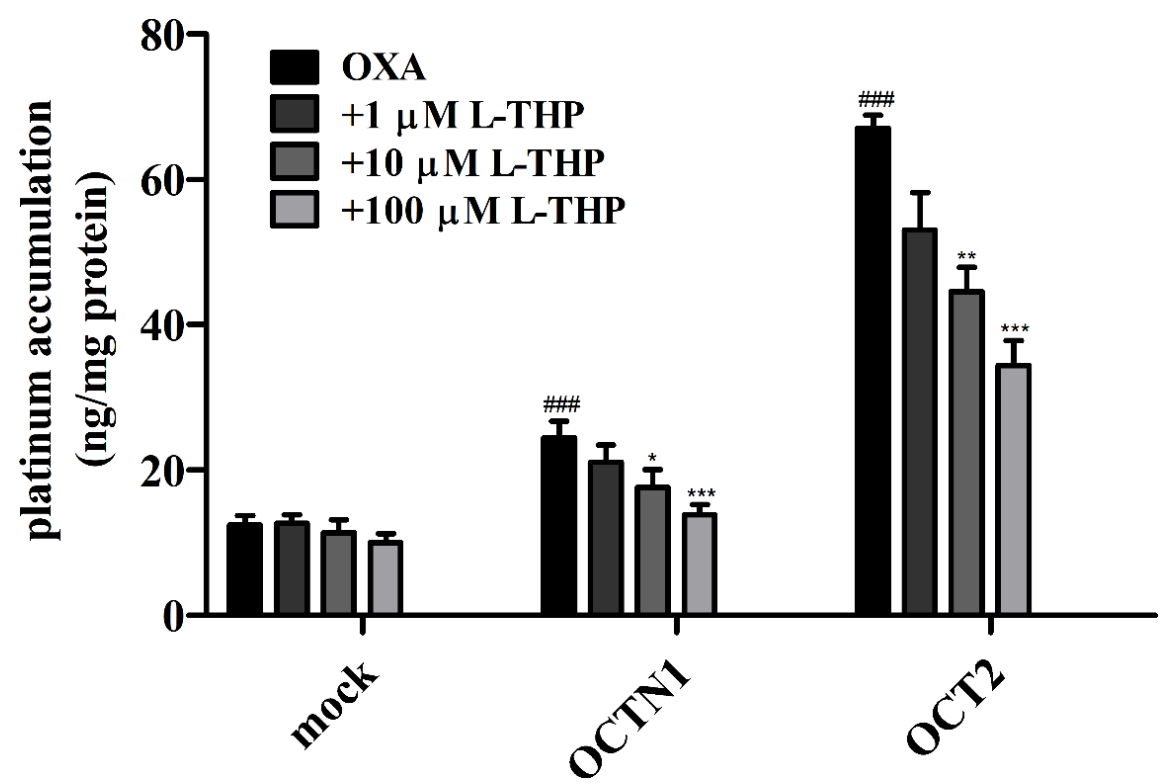

Figure 3. The effect of L-THP on the cellular accumulation of OXA in mock, MDCK-hOCTN1, and MDCK-hOCT2 cells. In the accumulation study, the cells were incubated with OXA ( $40 \mu \mathrm{M})$ in the absence or presence of L-THP for 5 min. Data are expressed as the mean \pm SEM, $\mathrm{n}=5$. Compared with the mock cells, ${ }^{\# \# \# ~} P<0.001$; compared with the OXA-alone group, ${ }^{*} P<0.01$ and $^{* *} P<0.01,{ }^{* * *} P<0.001$.

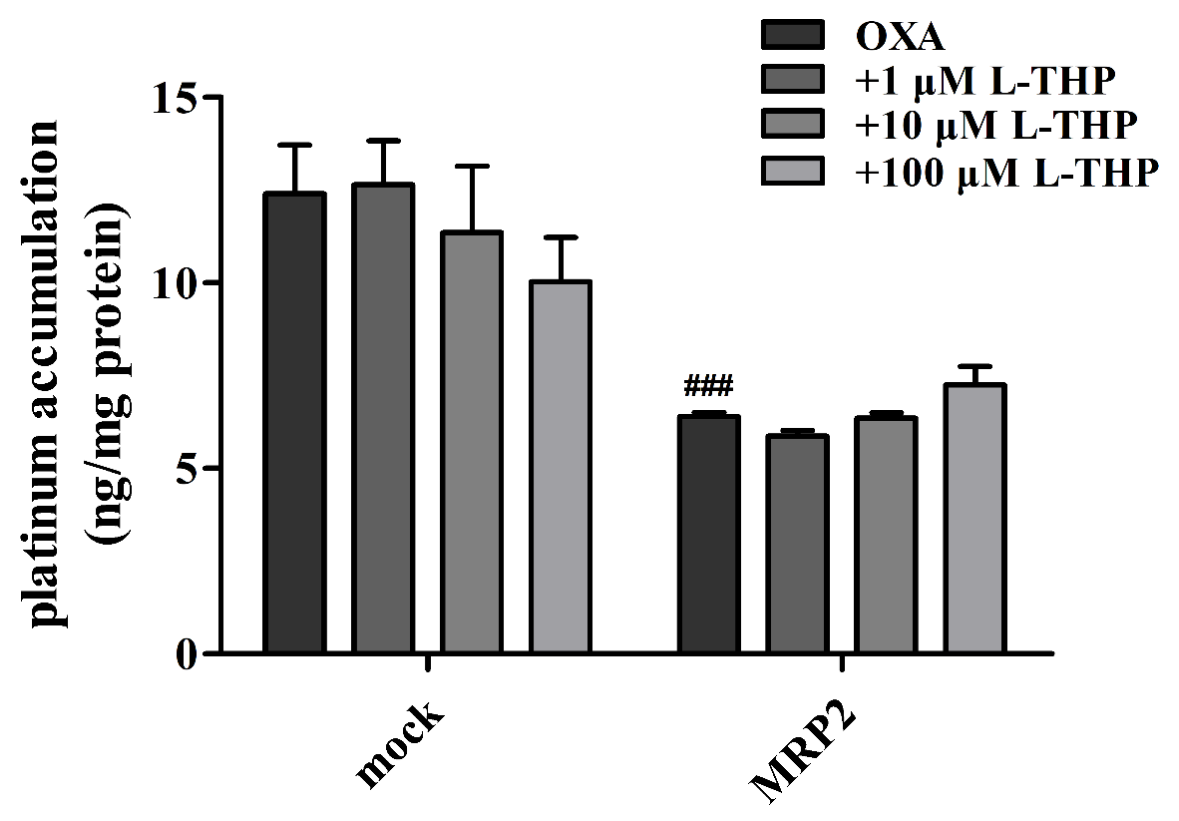


Figure 4. The effect of L-THP on the cellular accumulation of OXA in mock and MDCK-hMRP2 cells. The cells were incubated with OXA $(40 \mu \mathrm{M})$ in the absence or presence of L-THP. The data are expressed as the mean \pm SEM, $\mathrm{n}=5$; compared with the mock group, ${ }^{\# \# ~} \mathrm{P}<0.001$.
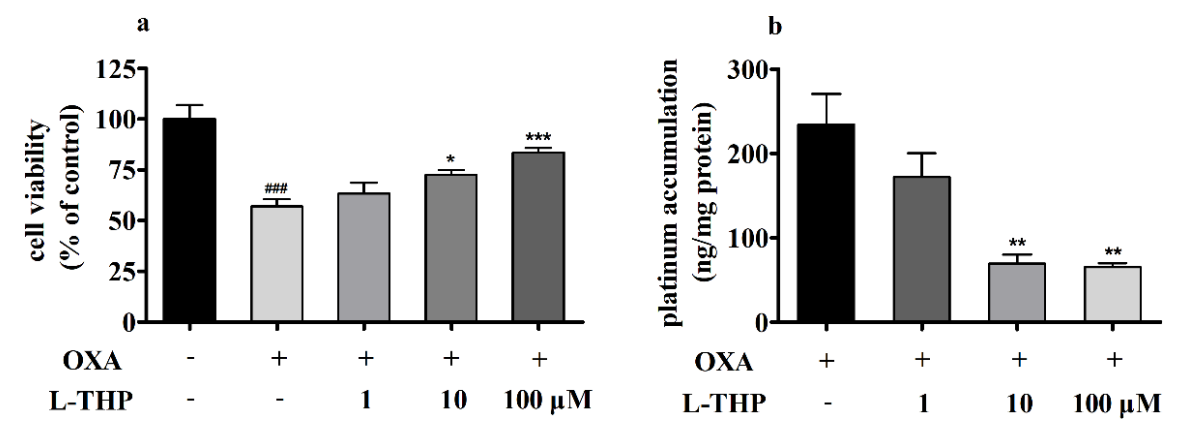

Figure 5. The effect of L-THP on cytotoxicity (a) and cellular accumulation (b) of OXA in primary DRG cells. Cells were incubated without or with OXA $(40 \mu \mathrm{M})$ and/or L-THP for $48 \mathrm{~h}$ in the cytotoxicity study and for $5 \mathrm{~min}$ in the accumulation study. Data are expressed as the mean $\pm \mathrm{SEM}, \mathrm{n}=6$ for the cytotoxicity study and $\mathrm{n}=5$ for the platinum accumulation study. Compared with the vehicle group, $\# \# \# P<0.001$; compared with the OXA-alone group, ${ }^{*} P<0.05,{ }^{* *} P<0.01$, and ${ }^{* * *} P<0.001$.
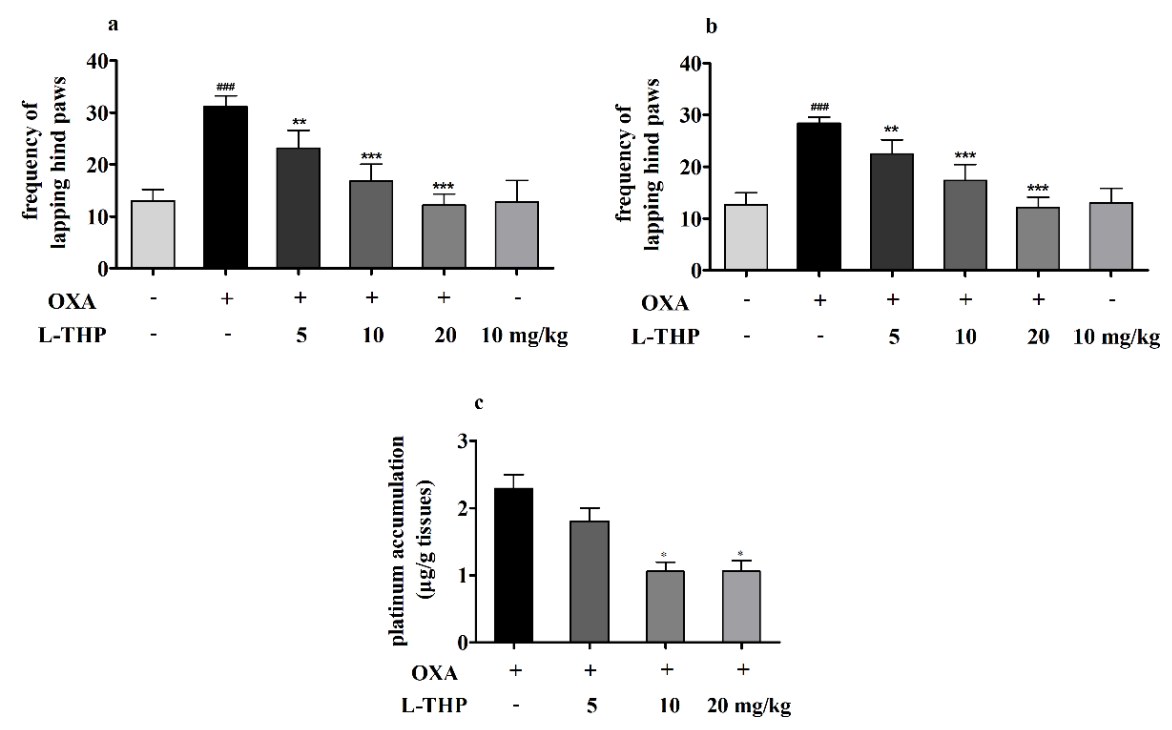

Figure 6. The effect of L-THP on OXA-induced frequency of lapping hind paws increases in male mice (a), female mice (b) and OXA accumulation in the DRG in gender matched mice (c). Data are expressed as the mean $\pm \mathrm{SEM}, \mathrm{n}=8$. Compared with the vehicle group, \#\#\# $P<0.001$; compared with the OXA alone group, ${ }^{*} P<0.05{ }^{* *} P<0.01$, and ${ }^{* * *} P<0.001$. 

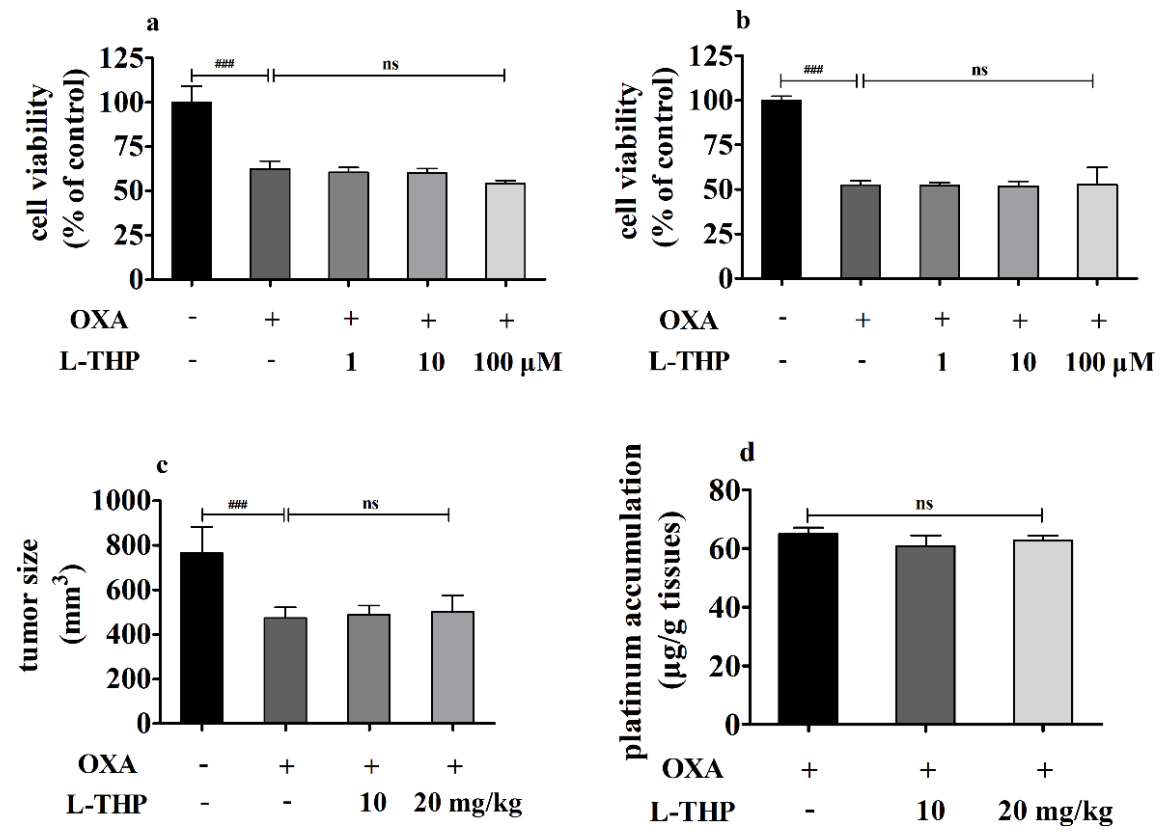

Figure 7. The effect of L-THP on the antitumor efficiency of OXA in HT29 (a) and SW620 cells (b) and on antitumour efficiency (c) or OXA accumulation in tumours (d) in HT29 tumour-bearing nude mice. Data are expressed as the mean \pm SEM, $n=6$ for the study in cells and $n=8$ for that in nude mice. Compared with the vehicle group, $\# \# \#<0.001$.
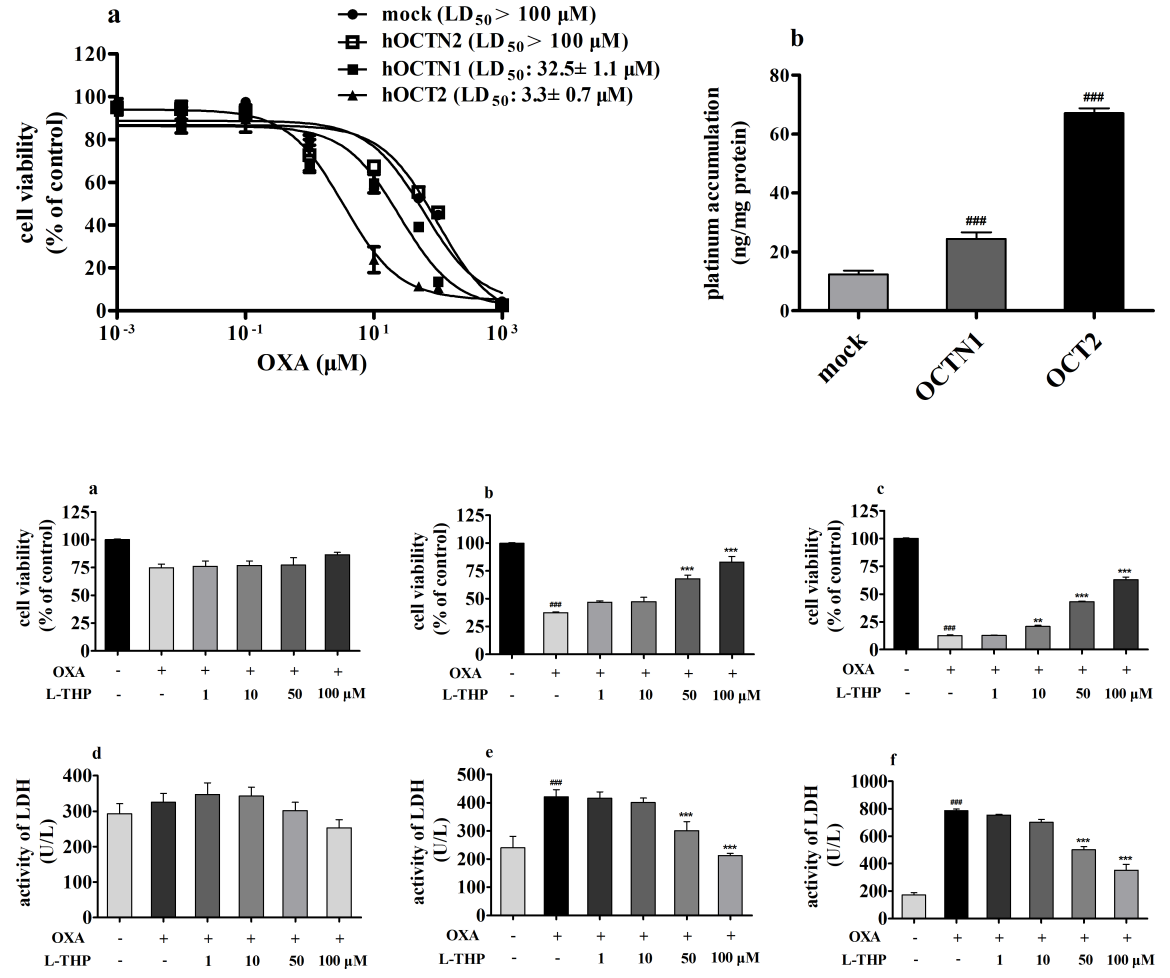

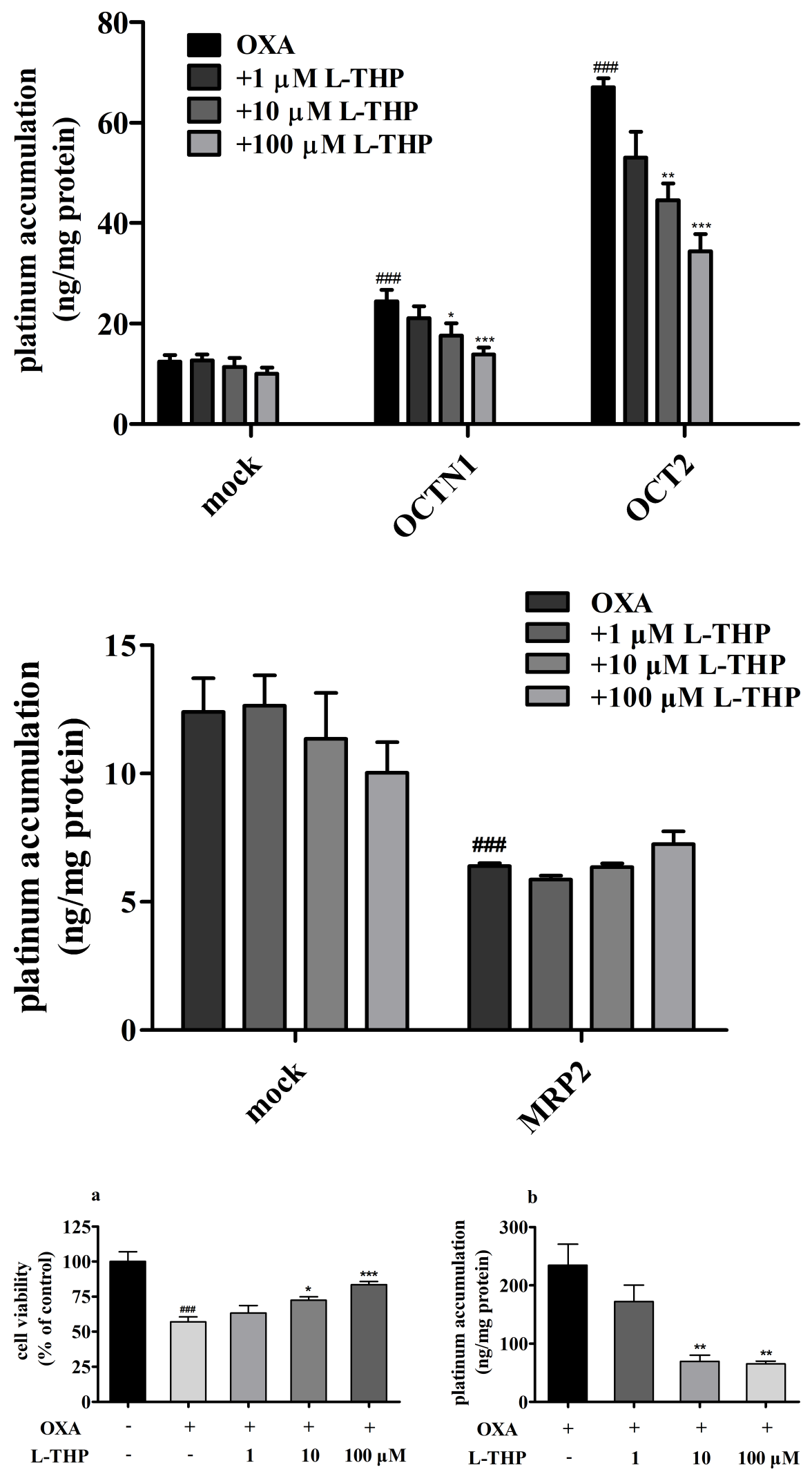

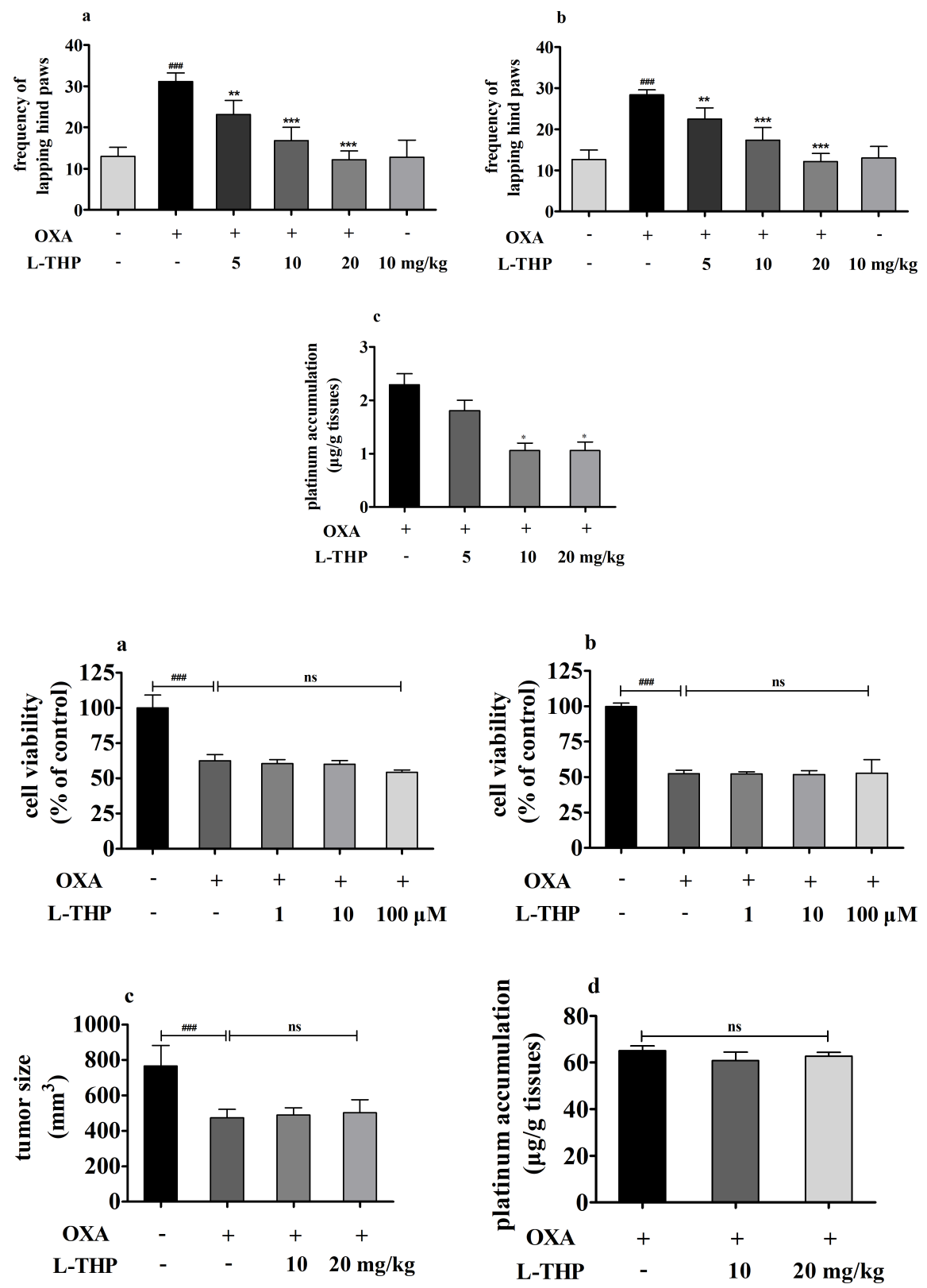